\title{
The tango of immunotherapy and targeted therapy in metastatic renal cell carcinoma
}

\author{
Nityam Rathi, Benjamin L. Maughan, Neeraj Agarwal, Umang Swami \\ Division of Medical Oncology, Department of Internal Medicine, Huntsman Cancer Insitute, University of Utah, Salt Lake City, UT, USA \\ Correspondence to: Neeraj Agarwal, MD. Professor of Medicine, Co-leader, HCI Experimental Therapeutics Program, Director, Genitourinary \\ Oncology Program, Huntsman Cancer Institute, University of Utah, 2000 Circle of Hope Drive Suite 5726, Salt Lake City, UT 84112, USA. Email: \\ neeraj.agarwal@hci.utah.edu; Umang Swami, MD, MS. Assistant Professor, Department of Oncology, Huntsman Cancer Institute, University of \\ Utah, 2000 Circle of Hope Drive Suite 5726, Salt Lake City, UT 84112, USA. Email: umang.swami@hci.utah.edu. \\ Comment on: Motzer RJ, Penkov K, Haanen J, et al. Avelumab plus Axitinib versus Sunitinib for Advanced Renal-Cell Carcinoma. N Engl J Med \\ 2019;380:1103-15.
}

Submitted Jun 26, 2019. Accepted for publication Jul 01, 2019.

doi: $10.21037 /$ tcr.2019.07.02

View this article at: http://dx.doi.org/10.21037/tcr.2019.07.02

Over the past decade, the mortality rate due to advanced renal cell carcinoma (RCC) has seen a significant decline (1). This has been accomplished mostly due to the rapid transformation of the treatment landscape of advanced clear cell RCC (ccRCC) which comprises $70 \%$ to $80 \%$ of RCC (2). Therapies targeting vascular endothelial growth factor pathway, mammalian target of rapamycin pathway, and immune checkpoints have replaced interferon- $\alpha$ and high dose interleukin-2 in clinical practice (2). Despite of these advancements, many patients do not benefit from many of these recent drug approvals. The attrition rate of patients from first line to second-line therapy is very high at $50 \%$, and only approximately $25 \%$ patients remain alive to allow treatment in the third line therapy setting (3). This has pushed the momentum to bring the most effective therapies to the first-line setting to improve outcomes. One recent strategy to achieve this has been to combine targeted therapies with immunotherapies.

The JAVELIN Renal 101 trial (4) is a randomized phase 3 study that compared avelumab plus axitinib with standard of care sunitinib in patients with advanced RCC. This study enrolled previously untreated advanced RCC patients with clear-cell histology component. The original primary objective was to show the superiority of avelumab plus axitinib in prolonging progression-free survival (PFS) in the intent-to-treat population, regardless of programmed death ligand 1 (PD-L1) status; however, a later protocol amendment changed the primary objective to show the superiority of avelumab plus axitinib over sunitinib with respect to PFS and overall survival (OS) in patients with PD-L1 positive tumors [defined as $\geq 1 \%$ of immune cells staining within tumor area by Ventana PD-L1 (SP263) assay]. Avelumab plus axitinib demonstrated a superior PFS as compared to sunitinib both in patients with PD-L1 positive tumors [13.8 vs. 7.2 months; hazard ratio (HR) $0.61 ; 95 \%$ confidence interval $(\mathrm{CI}), 0.47$ to 0.79 ; $\mathrm{P}<0.001]$, and the intent-to-treat population $(13.8$ vs. 8.4 months, HR 0.69; 95\% CI, 0.56 to $0.84 ; \mathrm{P}<0.001)$. The data for median OS is not mature yet. The rate of all grade and grade 3 or higher adverse events were similar in both arms (Table 1). Overall, $38.2 \%$ of patients on avelumab plus axitinib arm experienced immune-related adverse events, of which $9 \%$ were grade 3 or higher. A very small number of patients died due to immune related adverse events: $3(0.7 \%)$ patients in avelumab plus axitinib arm, due to treatment-related toxicities of sudden death, myocarditis, and necrotizing pancreatitis; while one $(0.2 \%)$ patient in the sunitinib arm died due to treatment-related toxicity of intestinal perforation. Results of this study have led to Food and Drug Administration approval of the combination as first-line therapy in patients with advanced RCC (9).

Similar to avelumab plus axitinib, multiple other agents and combinations such as ipilimumab with nivolumab (5), cabozantinib (6), pembrolizumab with axitinib (7) and atezolizumab with bevacizumab (8) have been compared to standard of care sunitinib in phase II and III trials with encouraging results. Table 1 provides a summary of these trials. Results of these studies have led to the inclusion of 


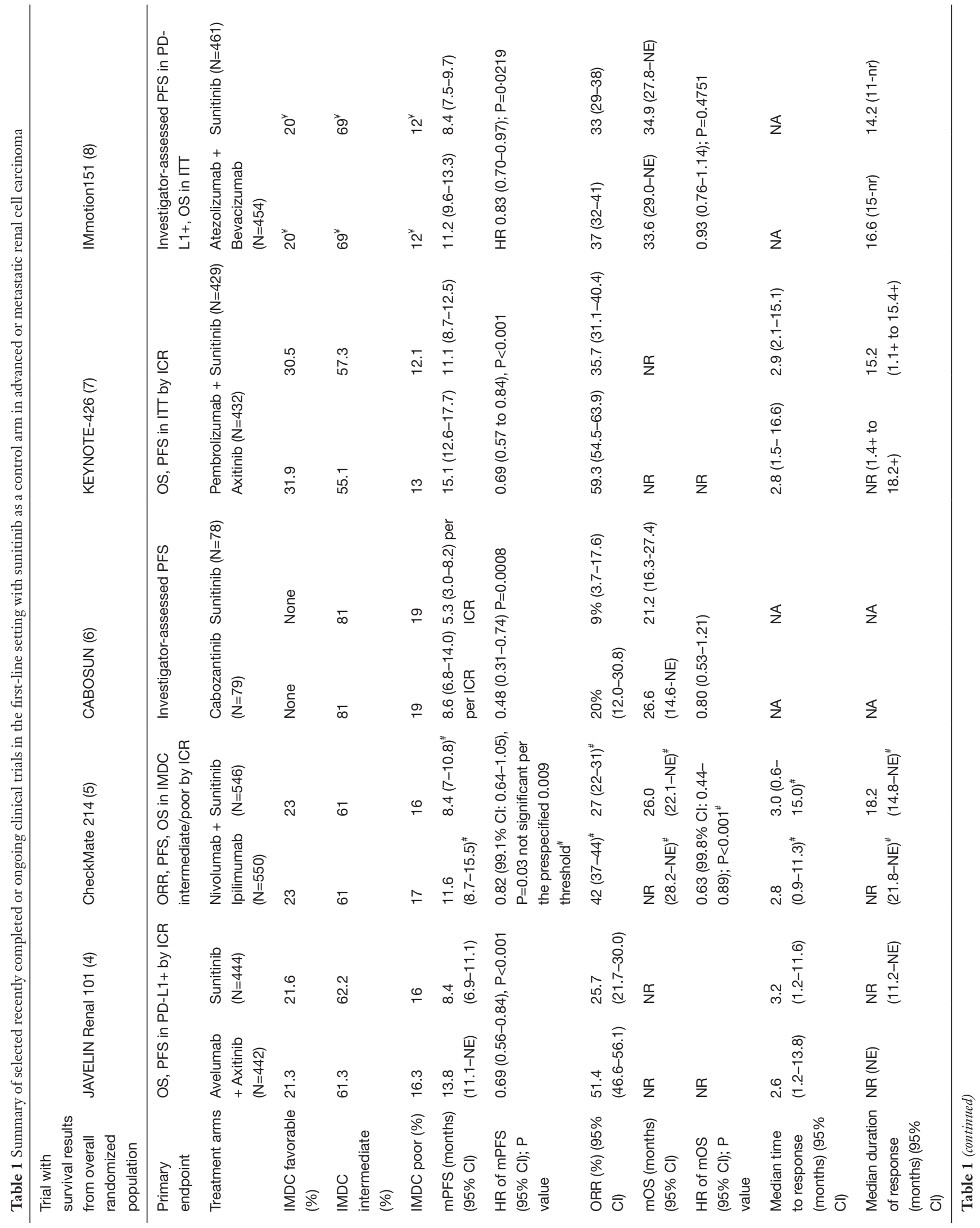




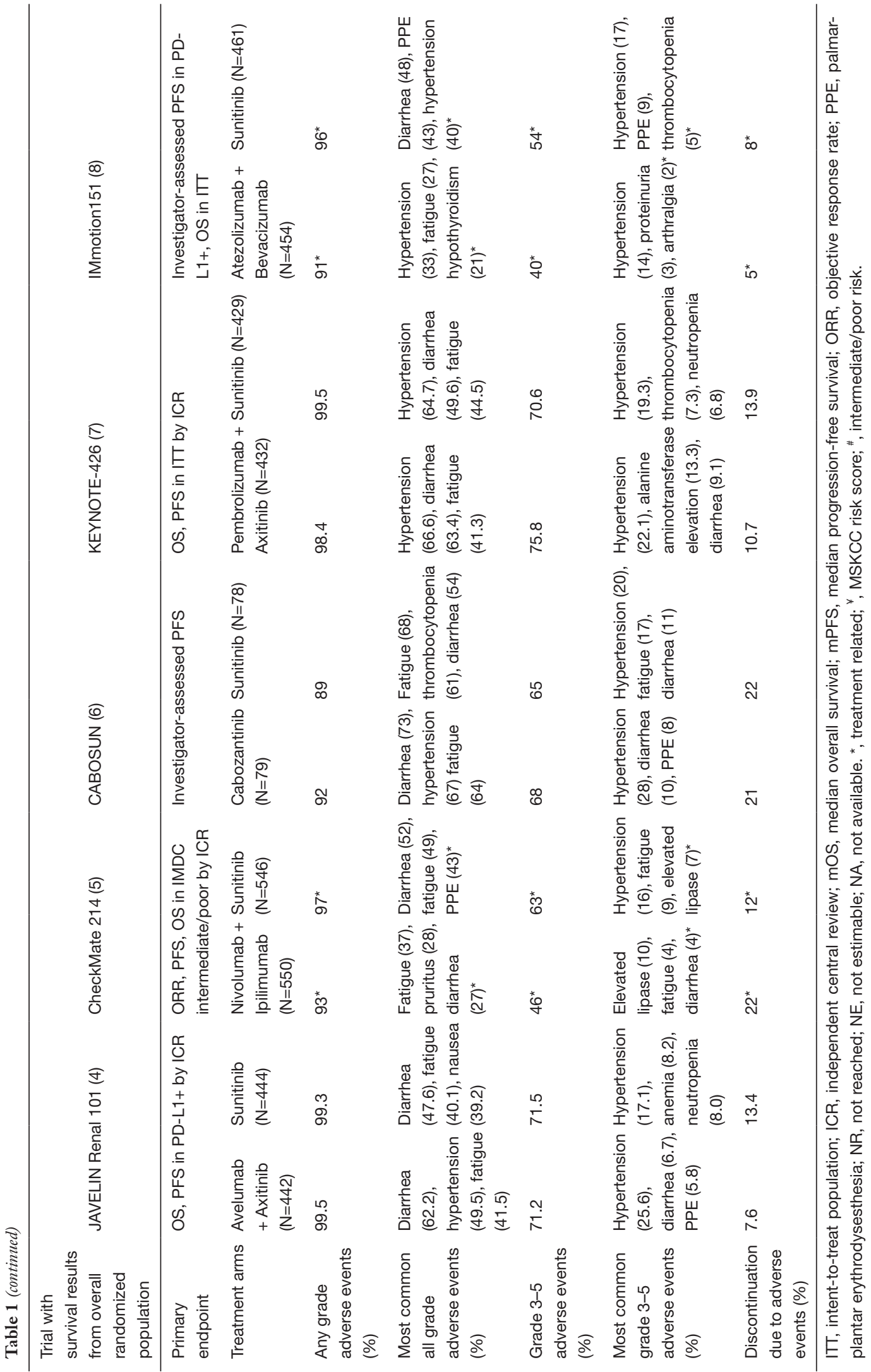


axitinib plus pembrolizumab as a preferred regimen, and avelumab with axitinib as another recommended regimen for all advanced ccRCC regardless of risk category in National Comprehensive Cancer Network (NCCN) guidelines (10). Ipilimumab plus nivolumab is designated as another recommended regimen for favorable risk, and as a preferred regimen along with cabozantinib for intermediate/poor IMDC risk RCC (10).

However, some questions remain unanswered. First, the best front-line combination is not yet defined. These above-mentioned trials had different patient populations, end-points and results that need to be understood (Table 1). As compared to sunitinib, avelumab with axitinib has shown improved PFS, and pembrolizumab with axitinib has shown improved PFS and OS in untreated advanced ccRCC patients $(4,7)$. This benefit (or trend towards benefit) has been observed across all IMDC and PD-L1 subgroups $(4,7,11)$. On the other hand, the nivolumab plus ipilimumab combination and cabozantinib have shown OS advantage as compared to sunitinib in intermediate and poor risk advanced ccRCC patients only $(5,6)$. In favorable risk patients, nivolumab plus ipilimumab as compared to sunitinib did not show an improved median PFS (13.9 vs. 19.9 months; HR 1.23 ; 95\% CI, 0.90-1.69; $\mathrm{P}=0.189$ ) or OS at 24 months ( $85 \%$ vs. $88 \%$ ) (12). When compared to sunitinib, atezolizumab with bevacizumab demonstrated a superior median PFS in the PD-L1 positive population (11.2 versus 7.7 months; HR 0.74; 95\% CI, 0.57-0.96; $\mathrm{P}=0.0217)$ while median $\mathrm{OS}$ in intent to treat population was similar (33.6 versus 34.9 months; HR 0.93 ; 95\% CI, 0.76-1.14; $\mathrm{P}=0.4751)$; however, the results did not cross the significance boundary at the interim analysis (8).

Therefore, due to these differences, there is a need to identify patients who will benefit from combination immunotherapies (anti-CTLA-4 with anti-PD-1) versus immunotherapy with antiangiogenic/targeted agents versus single-agent targeted therapy. In the JAVELIN Renal 101 trial, gene expression signatures and somatic mutations as determined by whole-exome sequencing and RNA sequencing were able to differentiate therapyspecific outcomes (13). Relative to wild-type, mutations in CD1631L, PTEN, or DNMT1 significantly differentiated treatment arm-specific PFS. High angiogenesis gene expression signature showed a significantly improved PFS in the sunitinib arm, but was not able to differentiate PFS in the avelumab plus axitinib arm. In contrast, avelumab plus axitinib showed an improved PFS as compared to sunitinib in low-angiogenesis subset. Similarly, high effector T cell and inflamed $\mathrm{T}$ cell signatures showed prolonged PFS with avelumab plus axitinib as compared to sunitinib. In the avelumab plus axitinib arm, an improved PFS was seen in patients with a positive novel immune gene expression signature versus those who were negative (HR 0.63; 95\% CI, 0.46-0.86; $\mathrm{P}=0.004)$. The results were validated in an independent dataset of the JAVELIN Renal 100 study (HR, 0.46; 95\% CI, 0.20-1.05; $\mathrm{P}=0.064$ ) (13). Similarly, in the CABOSUN trial, patients with MET-positive tumors (defined by $\geq 50 \%$ tumor cells stained $2+$ or $3+$ for positive status) showed an increased median PFS with cabozantinib as compared to sunitinib (13.8 vs. 3 months; HR 0.32; 95\% CI, 0.16-0.63), while in MET-negative patients median PFS with cabozantinib and sunitinib appeared similar (6.9 vs. 6.1 months; HR 0.67; 95\% CI, 0.37-1.23) (7). These results demonstrate that unique predictive biomarkers to guide treatment may be developed by combining genomic signatures with clinical data and bioinformatics from these large registration trials. However, it will need extensive collaboration between industry, cooperative groups, and governmental agencies to achieve standardization across platforms which would be difficult to achieve.

The next question pertains to the underlying reasons for observed clinical benefit with immunotherapy and targeted therapy. It is not clear whether the PFS and OS benefit noticed with combination therapies in JAVELIN Renal 101 and Keynote-426 trials $(4,7)$ is due to an additive effect of axitinib, a real synergistic effect, or due to modulation of tumor biology by front-line immunotherapy which possibly led to a decrease in tumor growth rate. In the phase III AXIS trial, patients with prior cytokine treatment and no prior exposure to VEGF inhibitors had a significantly better median PFS of 12.1 months with axitinib as compared to 6.5 months with sorafenib (HR 0.464; 95\% CI, 0.318-0.676; $\mathrm{P}<0.0001$ ) (14). Whereas in patients with prior treatment with VEGF inhibitor like sunitinib, median PFS was 4.8 months with axitinib and 3.4 months with sorafenib (HR 0.741; 95\% CI, 0.573-0.958; P=0.0107) (14). Emerging data also indicates that first-line avelumab plus axitinib treatment can lead to longer median PFS with secondline therapy (11).

Additional questions of whether the second-line therapies, which most likely will be tyrosine kinase inhibitors, will be effective once a patient has progressed on immunotherapy and targeted therapy combinations, or what sequence should be used in the clinic still need to be answered. A small retrospective analysis has shown that subsequent use of tyrosine kinase inhibitors still retain activity after progression on combination tyrosine kinase 
inhibitor plus checkpoint inhibitor therapy (15). It is to be noted that current combination trials have shown benefit in comparison to sunitinib, but so has cabozantinib (Table 1). Therefore, after progression on combination therapies of VEGF TKIs plus PD-1/PD-L1 inhibitors in the first-line therapy seting, cabozantinib may be the optimal secondline theray. For third-line, in our view, lenvatinib plus everolimus may be the most optimal option. There is no data to support the use of a PD-1/PD-L1 inhibitor after progression on a prior PD-1/PD-L1 inhibior either alone or in a combination therapy regimen.

However, these recommendations and opinions might also change very soon as multiple other phase 3 trials are currently exploring novel combinations in advanced RCC. Some noteworthy ongoing trials in advanced RCC include pazopanib plus abexinostat versus pazopanib (NCT03592472), lenvatinib plus everolimus or lenvatinib plus pembrolizumab versus sunitinib (NCT02811861), cabozantinib with nivolumab and ipilimumab versus nivolumab and ipilimumab (NCT03937219), nivolumab plus cabozantinib versus sunitinib (NCT03141177), and NKTR-214 plus nivolumab versus investigator's choice of sunitinib or cabozantinib (NCT03729245).

Studies are also looking into how to optimize advanced RCC treatment. The OMNIVORE (NCT03203473) trial evaluates nivolumab alone in patients with advanced RCC with an addition of ipilimumab only in non-responders. On the other hand, the PDIGREE (NCT03793166) trial is investigating the treatment approach of nivolumab and ipilimumab followed by nivolumab versus cabozantinib with nivolumab in patients with metastatic RCC, depending on response.

Another unanswered question is how long to continue immunotherapy-based combination treatment after responses. Prior experience with high dose interleukin-2 has shown that immunotherapy can lead to durable responses in advanced RCC even off of therapy (16). Ipilimumab with nivolumab has shown a significantly longer treatmentfree survival as compared to sunitinib in the CheckMate 214 trial (17). Therefore, it can be reasonably believed that most responses with PD-1/PD-L1 based immunotherapy combinations will also be durable (Table 1). Future investigations might evaluate treatment interruptions in responders, considering the durable nature of most responses, which can help alleviate not only treatmentrelated toxicities but also financial toxicities. At the same time, overcoming resistance in non-responders should be investigated as a priority as immunotherapy offers the potential of long-term remission or as some may say "cure", which is lacking in targeted therapies. Another field of unmet need is RCC with non-clear cell histology where strong clinical evidence and trial portfolios are lacking. We are encouraged by some progress with the non-clear cell histologies though. The SWOG 1500 clinical trial (NCT02761057) will define the standard of care first-line treatment for papillary RCC between 4 tyrosine kinase inhibitors cabozantinib, crizotinib, savolitinib, and sunitinib. Also, cabozantinib in combination with ipilimumab and nivolumab is being formally evaluated in non-clear cell RCC subtypes (NCT03866382).

To summarize, over last two years extensive and exciting progress with immunotherapies and targeted therapies has been achieved in advanced RCC. These therapies have proven to be very effective, but toxicities remain a concern and most patients still progress within a year. There is a continued need to improve on these fronts with more effective agents and innovative trials, especially in the second-line setting, along with the establishment of biomarkers that identify patients who can derive the most benefit from these treatments.

\section{Acknowledgments}

Funding: None.

\section{Footnote}

Provenance and Peer Review: This article was commissioned by the Editorial Office, Translational Cancer Research. It has not been sent for external peer review.

Conflicts of Interest: All authors have completed the ICMJE uniform disclosure form (available at http:// dx.doi.org/10.21037/tcr.2019.07.02). Benjamin Maughan is on consulting/advisory boards of Tempus, Peloton Therapeutics, Janssen Oncology, BMS, Astellas and Bayer; and institutional research funding from BMS and BavarianNordic. Neeraj Agarwal (career wide/past or present) reports consultancy/advisory fees from Pfizer, Novartis, Merck, Genentech, Eisai, Exelixis, Clovis, EMD Serono, Bristol-Myers Squibb, Astra Zeneca, Foundation One, Janssen, Astellas, Ely Lilly, Bayer, Argos, Medivation, Clovis, Pharmacyclics, and Nektar; and institutional research funding from Active Biotech, Astra Zeneca, Bavarian Nordic, Bayer, Bristol-Myers Squibb, Calithera, Celldex, Clovis, Eisai, Ely Lilly, EMD Serono, Exelixis, Genentech, GlaxoSmithKline, Immunomedics, Janssen, 
Medivation, Merck, Nektar, Newlink Genetics, Novartis, Pfizer, Prometheus, Rexahn, Sanofi, Takeda, and Tracon. Nityam Rathi and Umang Swami have no conflicts of interest to declare.

Ethical Statement: The authors are accountable for all aspects of the work in ensuring that questions related to the accuracy or integrity of any part of the work are appropriately investigated and resolved.

Open Access Statement: This is an Open Access article distributed in accordance with the Creative Commons Attribution-NonCommercial-NoDerivs 4.0 International License (CC BY-NC-ND 4.0), which permits the noncommercial replication and distribution of the article with the strict proviso that no changes or edits are made and the original work is properly cited (including links to both the formal publication through the relevant DOI and the license). See: https://creativecommons.org/licenses/by-nc-nd/4.0/.

\section{References}

1. Saad AM, Gad MM, Al-Husseini MJ, et al. Trends in Renal-Cell Carcinoma Incidence and Mortality in the United States in the Last 2 Decades: A SEER-Based Study. Clin Genitourin Cancer 2019;17:46-57.e5.

2. McKay RR, Bosse D, Choueiri TK. Evolving Systemic Treatment Landscape for Patients With Advanced Renal Cell Carcinoma. J Clin Oncol 2018:JCO2018790253.

3. Stukalin I, Wells JC, Fraccon A, et al. Fourth-Line Therapy in Metastatic Renal Cell Carcinoma (mRCC): Results from the International mRCC Database Consortium (IMDC). Kidney Cancer 2018;2:31-6.

4. Motzer RJ, Penkov K, Haanen J, et al. Avelumab plus Axitinib versus Sunitinib for Advanced Renal-Cell Carcinoma. N Engl J Med 2019;380:1103-15.

5. Motzer RJ, Tannir NM, McDermott DF, et al. Nivolumab plus Ipilimumab versus Sunitinib in Advanced Renal-Cell Carcinoma. N Engl J Med 2018;378:1277-90.

6. Choueiri TK, Hessel C, Halabi S, et al. Cabozantinib versus sunitinib as initial therapy for metastatic renal cell carcinoma of intermediate or poor risk (Alliance A031203 CABOSUN randomised trial): Progression-free survival by independent review and overall survival update. Eur J Cancer 2018;94:115-25.

7. Rini BI, Plimack ER, Stus V, et al. Pembrolizumab plus Axitinib versus Sunitinib for Advanced Renal-Cell Carcinoma. N Engl J Med 2019;380:1116-27.
8. Rini BI, Powles T, Atkins MB, et al. Atezolizumab plus bevacizumab versus sunitinib in patients with previously untreated metastatic renal cell carcinoma (IMmotion151): a multicentre, open-label, phase 3, randomised controlled trial. Lancet 2019;393:2404-15.

9. Food and Drug Administration. BAVENCIO ${ }^{\circledR}$ (avelumab) Prescribing Information. Available online: https://www.accessdata.fda.gov/drugsatfda_docs/ label/2019/761049s006lbl.pdf. Accessed June 10, 2019.

10. National Comprehensive Cancer Network. Kidney Cancer (Version 1.2020). Available online: https://www.nccn.org/ professionals/physician_gls/pdf/kidney.pdf. Accessed June 10, 2019.

11. Choueiri TK, Motzer RJ, Campbell MT, et al. Subgroup analysis from JAVELIN Renal 101: Outcomes for avelumab plus axitinib $(A+A x)$ versus sunitinib $(S)$ in advanced renal cell carcinoma (aRCC). J Clin Oncol 2019;37:abstr 544.

12. Tannir NM, Frontera OA, Hammers HJ, et al. Thirtymonth follow-up of the phase III CheckMate 214 trial of first-line nivolumab + ipilimumab $(\mathrm{N}+\mathrm{I})$ or sunitinib $(\mathrm{S})$ in patients (pts) with advanced renal cell carcinoma (aRCC). J Clin Oncol 2019;37:abstr 547.

13. Choueiri TK, Albiges L, Haanen JBAG, et al. Biomarker analyses from JAVELIN Renal 101: Avelumab + axitinib $(A+A x)$ versus sunitinib $(S)$ in advanced renal cell carcinoma (aRCC). J Clin Oncol 2019;37:abstr 101.

14. Rini BI, Escudier B, Tomczak P, et al. Comparative effectiveness of axitinib versus sorafenib in advanced renal cell carcinoma (AXIS): a randomised phase 3 trial. Lancet 2011;378:1931-9.

15. Ged Y, Gupta R, Duzgol C, et al. Systemic therapy for advanced clear cell renal cell carcinoma (ccRCC) after progression on immune-oncology plus VEGF targeted therapy combinations (IO-VEGF). J Clin Oncol 2019;37:abstr 4576.

16. Yang JC, Childs R. Immunotherapy for renal cell cancer. J Clin Oncol 2006;24:5576-83.

17. McDermott DF, Rini BI, Motzer RJ, et al. Treatment-free survival (TFS) after discontinuation of first-line nivolumab (NIVO) plus ipilimumab (IPI) or sunitinib (SUN) in intention-to-treat (ITT) and IMDC favorable-risk patients (pts) with advanced renal cell carcinoma (aRCC) from CheckMate 214. J Clin Oncol 2019;37:abstr 564.

Cite this article as: Rathi N, Maughan BL, Agarwal N, Swami U. The tango of immunotherapy and targeted therapy in metastatic renal cell carcinoma. Transl Cancer Res 2019;8(8):E1-E6. doi: 10.21037/tcr.2019.07.02 